\title{
The Effect of Low Dose Ketamine on the Need for Morphine in Patients with Multiple Trauma in Emergency Department
}

\author{
(1) Farhad Heydari, (1) Reza Azarian, (1) Babak Masoumi, (1) Alireza Abootalebi Ghahnavieh
}

Department of Emergency Medicine, School of Medicine, Isfahan University of Medical Sciences, Isfahan, Iran

\begin{abstract}
Aim: There are a few analgesic drugs (morphine, ketamine, Paracetamol, etc.) used for pain control in injury patients. Previous studies showed that low-dose ketamine (doses less than $1 \mathrm{mg} / \mathrm{kg}$ ) promptly and satisfactorily resolved pain. This study aimed to compare the analgesic effects of morphine with placebo (MP group) to that of a combination of morphine with ketamine (MK group) for patients with multiple trauma (MT) in the emergency department (ED).

Materials and Methods: This randomized, controlled, double-blinded clinical trial was conducted in the ED of two university teaching hospitals. Seventy multiple trauma patients aged between 16 and 50 years with a severe acute pain defined as a visual analog scale (VAS) score of at least 70 (between 0 and 100) were enrolled. Patients were randomly assigned to receive intravenous morphine $(0.1 \mathrm{mg} / \mathrm{kg})$ with placebo or morphine $(0.05 \mathrm{mg} / \mathrm{kg})$ with low-dose ketamine $(0.3 \mathrm{mg} / \mathrm{kg})$ for pain control. The pain intensity was evaluated before and $10^{\text {th }}$, $20^{\text {th }}, 30^{\text {th }}$, and $60^{\text {th }}$ minutes after the intervention, and probable side effects were recorded. Efficient analgesia was defined as a VAS score not exceeding 30. If the VAS score was still above 30 , additional morphine (3 mg) was administered for both groups. The primary outcome was the VAS score at 30 minutes.

Results: Seventy patients were enrolled in this study. There was no difference between the groups in terms of demographic characteristics or vital signs and baseline pain scores. The pain intensity decreased significantly in both groups 30 minutes after the intervention. However, there was no significant difference between the mean VAS scores of the two groups before and after the intervention. Morphine consumption was significantly lower in the MK group versus the MP group (9.3 \pm 2.2 vs $6.1 \pm 2.7, p=0.01)$. In addition, there was no significant difference between the two groups in terms of complications ( $p>0.05)$.

Conclusion: Low-dose ketamine in combination with morphine significantly reduced the need for an additional dose of morphine without increasing the complication rate related to morphine alone.
\end{abstract}

Keywords: Analgesic, emergency department, ketamine, morphine, multiple trauma, pain

\section{Introduction}

Pain is one of the most frequent problems in patients visiting emergency departments (EDs) so that $70 \%$ of emergency visits are due to pain (1-3). Adequate analgesia following trauma is a central aspect of emergency medical treatment before and after hospital admission (4). Acute pain and trauma are often closely related to one another, as pain is induced by noxious stimuli at the site of tissue damage (1). Pain is also the main complaint of patients seeking help in emergency care. As a consequence, patients suffer pain unnecessarily, and adverse physiological and psychological effects occur (2). Trauma patients, report low satisfaction with their pain management (5). In addition, trauma patients who present with multiple injuries, substance abuse, delayed care, as well as psychological and emotional issues complicate the care process $(5,6)$. Improved pain management is not only the patient right, but also it prompts early healing, reduces patient's stress response, shortens hospital length of stay, lowers costs, diminishes risk of chronic pain due to neuroplasticity, and ultimately reduces morbidity and improve long-term outcomes (5-8). 
There are several pain control medications (morphine, ketamine, paracetamol, etc.) for trauma patients. Opioid analgesics are commonly administered and prescribed from the ED. Morphine is the first choice in the treatment of severe nociceptive pain. $(3,5)$. Owing to its few complications and relatively rapid and effective analgesic effects, it is widely administered $(5,8,9)$. However, adverse effects of opioids are nausea, vomiting, dizziness, constipation, reduced blood pressure, reduced heart rate, and reduced oxygen saturation. Sometimes these complications occur before sufficient pain relief (5-10). Ketamine is an N-methyl-Daspartate receptor antagonist, which has been utilized since the 1970s for anesthesia (8). Ketamine comparably potentiate opioid analgesia, and reducing opioid need in postoperative settings (11). Adverse effects include increased secretions, blurred vision, agitation on emergence, elevated pulse and blood pressure, disturbed sleep and hallucinations (5-8,12). Low dose ketamine, by definition, is applied to doses less than $1 \mathrm{mg} / \mathrm{kg}$ (13). Low dose ketamine provides analgesic effectiveness and apparent safety comparable to that of intravenous morphine for short-term treatment of acute pain in the $\operatorname{ED}(12,13)$ and in trauma patients with severe acute pain reduces morphine requirements (14).

Various dosage protocols for morphine have been developed, producing contradictory results (1, 9, and 15). For example, Bijur et al. (9) conducted a study on 119 patients with acute and severe pain and found that approximately $67 \%$ of the patients receiving normal dosage $(0.1 \mathrm{mg} / \mathrm{kg})$ reported a reduction of pain of less than $50 \%$. They concluded that this dosage is insufficient for pain control. Although there is no standard dose for morphine, the majority of references have used a single dose of $0.1 \mathrm{mg} / \mathrm{kg}$, or $0.05-0.15 \mathrm{mg} / \mathrm{kg}(15,16)$.

There are few studies comparing the analgesic effect of morphine alone with the morphine-ketamine combination. To the best of our knowledge, this is the first study that compared the combination of intravenous Low dose ketamine at a dose of 0.3 $\mathrm{mg} / \mathrm{kg}$ with morphine at a dose of $0.05 \mathrm{mg} / \mathrm{kg}$ in trauma patients with a large sample size.

We intended to test the hypothesis that the combination of low dose ketamine with morphine would promptly reduce pain perception and morphine consumption compared with morphine alone in multiple trauma patients with severe acute pain at the ED.

\section{Materials and Methods}

\section{Study Design and Participants}

This prospective, randomized, double-blinded clinical trial was conducted in the adult ED of Isfahan University, two university educational hospitals, affiliated with Isfahan University of
Medical Sciences in Iran from December, 2016 to December, 2017. The study protocol was approved by the ethics committee of Isfahan University of Medical Sciences (IR.MUI.REC.1395.3.824). The trial was registered in the Iranian Registry of Clinical Trials under the number (IRCT20180129038549N3). Before the study, written informed consent was obtained from all parents, before enrolment into the study.

All the patients between 16 to 50 years with multiple trauma referring to ED were eligible for inclusion. Patients with a severe acute pain defined as a visual analog scale (VAS) $(5,14)$ score of at least 70/100; and without acute respiratory, hemodynamic, or neurologic compromise (respiratory distress signs, heart rate lower than 60, and systolic blood pressure lower than $90 \mathrm{~mm}$ $\mathrm{Hg}$, Glasgow Coma Score less than 15) were enrolled in this study.

Exclusion criteria were patients with psychiatric illness; history of cardiac disease; chronic respiratory, renal, or hepatic failure; known allergy to morphine or ketamine; treatment of chronic pain or treatment with opioids; incapacity to understand the VAS; pregnant or breast-feeding and presence of acute ocular or head trauma. Patients who had already received an opioid analgesic were also excluded.

The participants were randomly allocated to two groups using a statistical software to ensure roughly equal numbers in each group.

Pain measurement was accomplished using a $100-\mathrm{mm}$ ruler with tick marks spaced 10-mm apart that the patient points to the point indicating his or her pain intensity. The patient's pain intensity was represented by a point between " 0 " and means "no pain at all" and "100" and means "the worst pain imaginable".

\section{Intervention}

Eligible patients (70) were randomly allocated to receive either morphine and placebo (MP group) or morphine and ketamine (MK group), each with 35 patients. The first group (MP group) received $0.1 \mathrm{mg} / \mathrm{kg}$ intravenous morphine with $5 \mathrm{cc}$ isotonic sodium chloride solution as placebo for pain control. The second group (MK group) received $0.05 \mathrm{mg} / \mathrm{kg}$ intravenous morphine with $0.3 \mathrm{mg} / \mathrm{kg}$ intravenous ketamine (Ketamine hydrochloride $50 \mathrm{mg} / \mathrm{mL}$ vials, Rotexmedica, Trittau, Germany). Ketamine and placebo were administered from syringes of similar appearances prepared by a nurse who was otherwise not involved in the study. In addition, morphine in the second group was diluted to obtain an equal volume as the first group. The medications were administered intravenously by a nurse who was blinded to the study protocol.

Patients were asked to assess the intensity of their pain level based on VAS score on inclusion (T0) and then every 5 minutes 
after arrival at hospital. The patients' VAS scores were recorded at $0^{\text {th }}, 10^{\text {th }}, 20^{\text {th }}, 30^{\text {th }}$ and $60^{\text {th }}$ minutes after intervention. Sufficient pain reduction was obtained as defined by a VAS score, not exceeding $30 \%$ or $50 \%$ below the initial score. If the pain relief was not obtained, at those moments, additional morphine dose (3 mg) every 10 minutes was administered for each group. The safety evaluation included non-invasive monitoring of blood pressure, heart rate, oxygen saturation by pulse oximetry $\left(\mathrm{Spo}_{2}\right)$, and respiratory rate, at these periods. The presence of adverse effects was likewise recorded. These data were recorded at T0 and T30.

Sixty minutes after the first injection (T60), patients' satisfaction regarding analgesia (pain relief classified as excellent, good, mild, or weak) was recorded. Morphine consumption at T60 was recorded too.

The primary outcome was VAS at 30 minutes and morphine consumption and side effects were the secondary outcomes.

\section{Statistical Analysis}

Data were analyzed by the SPSS 22 software. Results were presented as the mean \pm standard deviation for quantitative variables. Pain scores were compared with independent t-test and qualitative variables were analyzed by the chi-square test except for patient satisfaction, which was analyzed by Fisher's exact test. Power analysis determined that a sample size of at least 30 subjects per group would achieve $90 \%$ power to detect a VAS difference of more than 13/100 (13) between treatment groups, with an $\alpha$ level of 0.05 . We chose to include 35 patients in each group to increase the power of this study. A $p$ value of less than 0.05 was considered statistically significant.

\section{Results}

Between December 01, 2016, and December 30, 2017, seventy patients were enrolled in the study (Figure 1). Two and one patients were excluded because of unwillingness to receive an additional dosage of morphine and being severely restless, respectively. Thus, data from 67 patients were completed and analyzed, 33 in the MP group and 34 in the MK group. Patients' demographic characteristics are shown in Table 1. The patients' mean ages were $38.5 \pm 14.3$ and $37.0 \pm 12.5$ years in MK and MP groups, respectively. There was no difference between the groups in terms of demographic characteristics or vital signs and baseline pain scores.

In both groups, 30 minutes after injection (T30), the mean VAS score when compared to the initial VAS score (T0) had a statistically significant reduction $(p=0.01)$ (Figure 2$)$. This reduction was slightly higher in the MK group than the MP group (56 versus

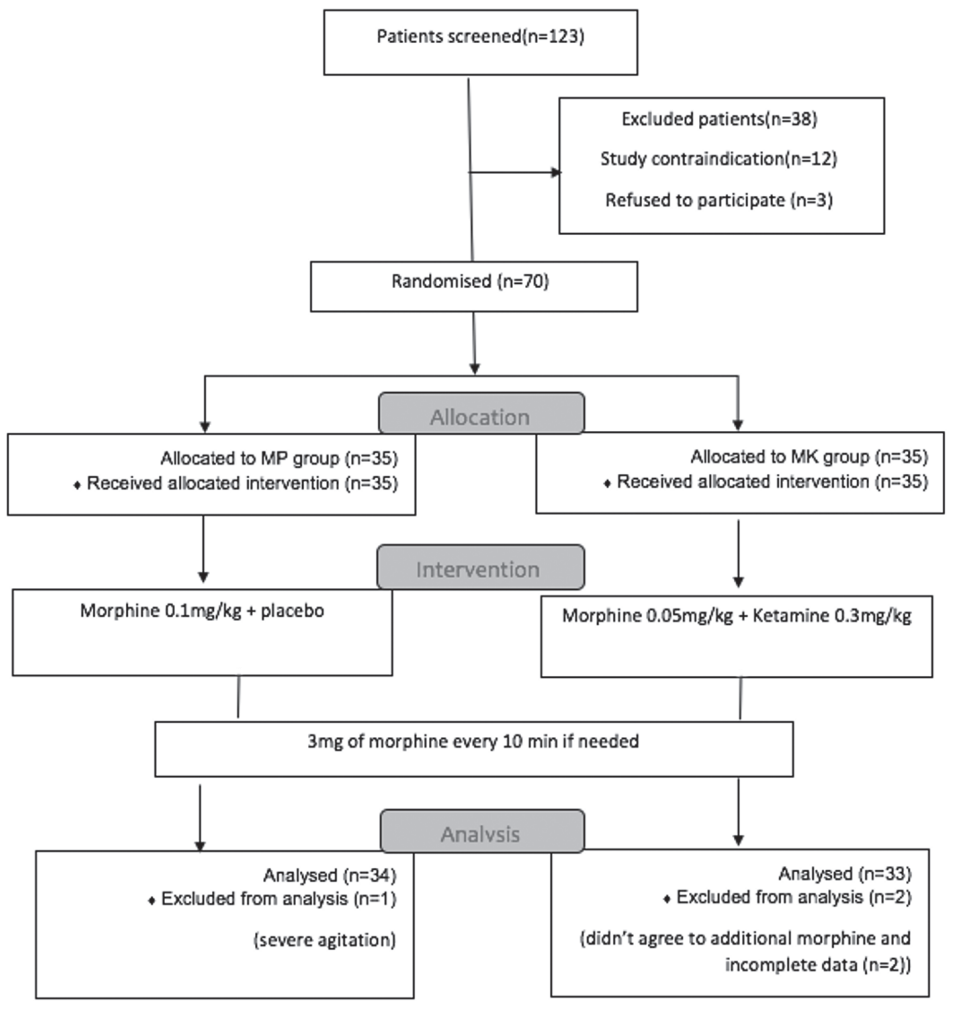

Figure 1. CONSORT flow diagram of the study

CONSORT: Consolidated Standards of Reporting Trials, MP: Morphine with placebo, MK: Morphine with ketamine, n: Number

Table 1. The baseline characteristics of patients from MK and MP groups

\begin{tabular}{|c|c|c|c|}
\hline Variables & $\begin{array}{l}\text { MP group, } \\
(n=33)\end{array}$ & $\begin{array}{l}\text { MK group, } \\
(n=34)\end{array}$ & $p$ value \\
\hline Age, mean (SD), year & $37.03 \pm 12.48$ & $38.47 \pm 14.28$ & 0.112 \\
\hline Sex ratio (male-female) & $25: 8$ & $24: 10$ & 0.611 \\
\hline Weight, kg & $78.3 \pm 7.1$ & $76.9 \pm 8.3$ & 0.534 \\
\hline \multicolumn{4}{|l|}{ Mechanism of injury } \\
\hline Road traffic accidents (\%) & $16(48.48)$ & 18 (52.94) & \multirow{4}{*}{0.831} \\
\hline Fall (\%) & $8(24.24)$ & $9(26.47)$ & \\
\hline Assault (\%) & $6(18.18)$ & 4 11.77) & \\
\hline Others (\%) & $3(9.10)$ & $3(8.82)$ & \\
\hline \multicolumn{4}{|l|}{ Final diagnosis, n (\%) } \\
\hline Fracture & $14(42.42)$ & $17(50.00)$ & \multirow{2}{*}{0.684} \\
\hline Soft tissue injury & $19(57.58)$ & $17(50.00)$ & \\
\hline
\end{tabular}

51 units); Moreover, the VAS score was not significantly different between MK and MP groups at T0, T10, T20, and T30 (Table 2).

According to the results, the VAS score at T30 was higher than 30 in 4 patients (6\%) in the MP group and 5 patients (7.5\%) in the MK group, representing a nonsignificant difference $(p=0.81)$. 
There were no differences between groups with regard to blood pressure, heart rate, or the respiratory rate at T0 and T30 (Table 3). Also, the oxygen saturation at T0 and T30 was not significantly different between groups; whereas, reduction in oxygen saturation was higher in the MP group. There was no clinically significant change of vital signs after enrolment.

No life-threatening complication was observed in both groups. There was no significant difference between groups in the incidence of adverse effects ( $p>0.05)$. The frequency of nausea

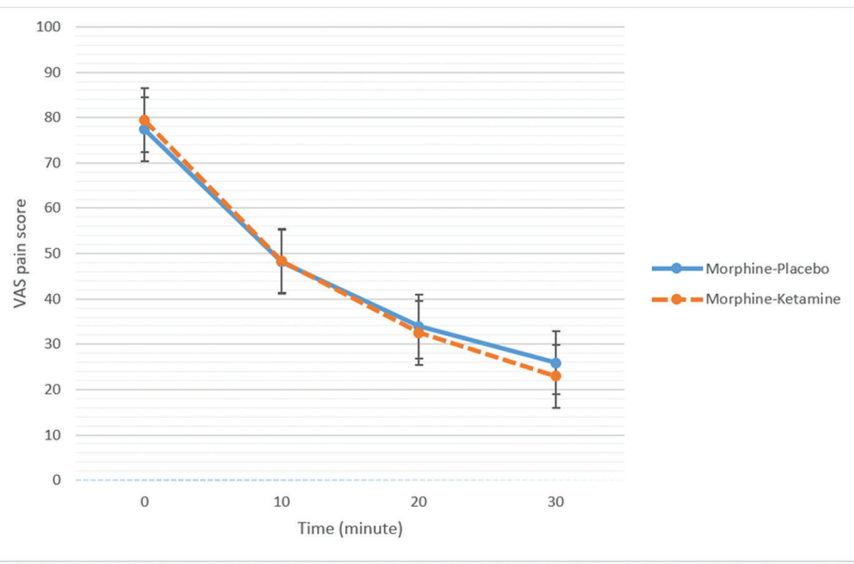

Figure 2. Mean score of pain before injection and $10^{\text {th }}, 20^{\text {th }}$, and $30^{\text {th }}$ minutes after it in both groups

VAS: Visual analog scale and vomiting was higher in the MP group than the MK group; whereas, the frequency of restlessness and delusion was higher in the MK group. However, this difference was not significant (Table 3).

Sixty minutes (T60) after injection, when the sedation effect of drugs has subsided, the patients' satisfaction was evaluated. A total of, 25 patients (75.7\%) in the MP group and 22 patients (64.7\%) in the MK group were satisfied with the analgesic (excellent and good). The satisfaction of patients was not significantly different between the two groups $(p=0.31)$ (Table 4$)$.

We divided the patients into two subgroups, with and without fractures. Fourteen patients (42.4\%) in the MP group and 17 patients $(50 \%)$ in the MK group did not have a fracture. There was a significant relationship between the lack of fracture and satisfaction of analgesics in both groups ( $p=0.01$ in MP group and $p=0.026$ in MK group). Also, in both subgroups, there was no significant difference between MP and MK groups regarding the patients' satisfaction $(p=0.668$ in with fracture and $p=0.236$ in without fracture subgroup).

The mean dose of morphine in MK and MP group was $9.3 \pm 2.2$ $\mathrm{mg}$ and $6.1 \pm 2.7 \mathrm{mg}$, respectively. At T60, morphine consumption was significantly lower in the MK group than that in the MP group, corresponding to significantly fewer morphine boluses in the MK group than those in the MP group (Table 4).

\begin{tabular}{|c|c|c|c|c|c|c|c|c|}
\hline \multirow[b]{2}{*}{ Time (minutes) } & \multicolumn{3}{|c|}{ MP group } & \multicolumn{3}{|c|}{ MK group } & \multirow{2}{*}{ b-a } & \multirow{2}{*}{ p value $e^{* *}$} \\
\hline & VAS score & Reduction in VAS score (a) & p value* & VAS score & Reduction in VAS score (b) & p value* & & \\
\hline 10 & 48.18 & 29.24 & $<0.001$ & 48.24 & 31.17 & $<0.001$ & 1.93 & 0.312 \\
\hline 20 & 33.93 & 43.49 & $<0.001$ & 32.50 & 46.91 & $<0.001$ & 3.42 & 0.111 \\
\hline
\end{tabular}

Table 3. Effect of treatment on clinical parameters in MP and MK groups

\begin{tabular}{|c|c|c|c|c|c|c|c|c|c|c|}
\hline \multirow[b]{2}{*}{ Parameter } & \multicolumn{4}{|c|}{ MP group } & \multicolumn{4}{|c|}{ MK group } & \multirow{2}{*}{ b-a } & \multirow{2}{*}{$p$ value $* *$} \\
\hline & T0 & T30 & Reduction (a) & p value* & T0 & T30 & Reduction (b) & p value* & & \\
\hline $\begin{array}{l}\text { Systolic blood pressure } \\
\text { (mm Hg, mean) }\end{array}$ & 124.21 & 113.06 & 11.15 & $<0.001$ & 128.47 & 118.52 & 9.95 & $<0.001$ & 1.2 & 0.565 \\
\hline $\begin{array}{l}\text { Heart rate } \\
\text { (beats/minute, mean) }\end{array}$ & 81.21 & 74.75 & 6.46 & $<0.001$ & 83.55 & 77.55 & 6.00 & $<0.001$ & 0.46 & 0.711 \\
\hline $\begin{array}{l}\text { Respiratory rate } \\
\text { (breaths/minute, mean) }\end{array}$ & 21.09 & 20.00 & 1.09 & $<0.001$ & 20.64 & 19.82 & 0.82 & $<0.001$ & 0.27 & 0.344 \\
\hline Pulse oximetry (\%, mean) & 98.45 & 94.63 & 3.82 & $<0.001$ & 98.79 & 98.02 & 0.77 & 0.056 & 3.05 & 0.302 \\
\hline \multicolumn{11}{|c|}{$\begin{array}{l}\text { *The } p \text { value for the reduction in parameters by } 30 \text { minutes within each group } \\
\text { **The } p \text { value for the contrast in the reduction between the } \\
\text { MK: Morphine with ketamine, } n \text { : Number, MP: Morphine with placebo }\end{array}$} \\
\hline
\end{tabular}




\begin{tabular}{|c|c|c|c|c|}
\hline \multicolumn{2}{|l|}{ Variables } & $\begin{array}{l}\text { MP group } \\
(n=33)\end{array}$ & $\begin{array}{l}\text { MK group } \\
(n=34)\end{array}$ & $p$ value \\
\hline \multirow{4}{*}{$\begin{array}{l}\text { Patient } \\
\text { satisfaction } \\
\text { T60 [n (\%)] }\end{array}$} & Weak & $1(3 \%)$ & 2 (5.9\%) & \multirow{4}{*}{0.311} \\
\hline & Mild & 7 (21.2\%) & 10 (38.2\%) & \\
\hline & Good & 18 (54.5\%) & 15 (44.1\%) & \\
\hline & Excellent & 7 (21.2\%) & $7(20.6 \%)$ & \\
\hline
\end{tabular}

Morphine consumption

\begin{tabular}{|l|l|l|l}
\hline T60 (mg, mean \pm SD) & $9.31 \pm 2.24$ & $6.14 \pm 2.74$ & 0.010
\end{tabular}

MP: Morphine with placebo, MK: Morphine with ketamine, n: Number, SD: Standard deviation

\section{Discussion}

In our study, we compared low dose ketamine plus morphine with morphine alone for ED multiple trauma patients experiencing acute severe pain. Our study suggests that low dose ketamine is as effective as morphine in relieving pain at $10^{\text {th }}, 20^{\text {th }}$ and $30^{\text {th }}$ minutes. Low dose ketamine has been shown to be safe and effective for the treatment of severe acute pain in the ED. We found that the association of low dose ketamine with reduced morphine consumption. At T60, morphine consumption was significantly lower in the MK group than that in the MP group. Significant reduction in pain intensity measured on a VAS at T30 was observed in both groups. This reduction was slightly higher in the MK group than the MP group; however, this difference was not significant. The side effects were also comparable between the two study groups.

Many studies have shown reductions in morphine requirements when ketamine is administered concomitantly $(12,14)$. Galinski et al. (14) performed the first study on the effect of morphine - low dose ketamine combination on trauma patients with acute and severe pain and have shown reductions in morphine requirements when ketamine is administered concomitantly.

There have been several published trials examining ketamine used for analgesia in ED patients. But there are few studies comparing the analgesic effect of morphine and combination of the morphine at a dose $0.05 \mathrm{mg} / \mathrm{kg}$ with low dose ketamine at a dose of $0.3 \mathrm{mg} / \mathrm{kg}$ in multiple trauma patients.

There are different doses of morphine, the majority of references have used a single dose of $0.1 \mathrm{mg} / \mathrm{kg}$, or $0.05-0.15 \mathrm{mg} / \mathrm{kg}(15,16)$. Bounes et al. (16) performed a study on 106 patients, who received either $0.05 \mathrm{mg} / \mathrm{kg}$ morphine then $0.025 \mathrm{mg} / \mathrm{kg}$, every 5 minutes or $0.1 \mathrm{mg} / \mathrm{kg}$ morphine then $0.05 \mathrm{mg} / \mathrm{kg}$ every 5 minutes intravenously. They have reported that the high-dose morphine regimen showed a similar analgesic response pattern to the low dose one in severe acute pain in a prehospital setting. But in the interests of achieving rapid pain relief, an initial dose of $0.05 \mathrm{mg} /$ $\mathrm{kg}$ should no longer be recommended for treating severe acute pain in a prehospital setting. So, we decided to demonstrate low dose ketamine with low dose morphine in trauma patients for pain control in the emergency setting.

Farsi et al. (15) were showed that using two doses of morphine instead of one is a safe and effective method for pain reduction in isolated limb trauma. They recommend performing a second injection of $0.05 \mathrm{mg} / \mathrm{kg}$ morphine 30 minutes after the initial standard dose of $0.10 \mathrm{mg} / \mathrm{kg}$ to decrease pain in these patients.

Like our study, Galinski et al. (14) found that the VAS pain measure was not statistically different between the morphine and ketamine intervention group and the morphine alone control group. At the 30-minute period, a larger proportion of the ketamine group had had their pain reduced to below $30 \mathrm{~mm}$ than the morphine group; however, this difference did not reach statistical significance.

In a study by Majidinejad et al. (17) ketamine $0.5 \mathrm{mg} / \mathrm{kg}$ was compared to morphine $0.1 \mathrm{mg} / \mathrm{kg}$ in 126 emergency patients with long bone fractures. Ketamine was found to have a significant decrease in the severity of acute pain at 10 minutes after the dose. This study reported that this onset of effect was similar to the morphine group. This study noted that adverse events were higher in the ketamine group but concluded that these complications should not preclude the use of ketamine as all effects resolved spontaneously without therapeutic intervention.

Miller et al. (18) performed a study on 45 patients, 21 patients received $0.1 \mathrm{mg} / \mathrm{kg}$ morphine and 24 patients received $0.3 \mathrm{mg} /$ $\mathrm{kg}$ ketamine. They showed low-dose ketamine did not produce a greater reduction in pain scores compared with morphine for acute pain in the ED. However, low-dose ketamine induced a significant analgesic effect within 5 minutes (15 minutes later the effect of ketamine was reduced) and provided a moderate reduction in pain for 2 hours. Adverse events, and nurse satisfaction scores were similar between groups. Our study and all three of these studies concurred that ketamine, although not superior to morphine, provides similar and significant pain reduction with a reasonably safe adverse effect profile $(14,17,18)$.

Several prospective randomized trials examined the analgesic effect of low dose ketamine and morphine combination on patients with acute severe pain. Their results were similar whatever their designs, namely, that low dose ketamine reduced morphine requirements and pain intensity $(11,12,14,19)$. Beaudoin et al. (19) evaluated two different doses of low dose ketamine-morphine combinations compared with morphine alone for ED analgesia showed a clinically significant decrease 
in pain intensity for more than $50 \%$ of patients who received morphine $(0.1 \mathrm{mg} / \mathrm{kg})$ and ketamine $(0.15$ or $0.3 \mathrm{mg} / \mathrm{kg}$ ) combination compared with the morphine-only group. In addition, the authors concluded that morphine combined with ketamine at a dose of $0.3 \mathrm{mg} / \mathrm{kg}$ had more efficacious analgesic effect than a combination using a ketamine dose of $0.15 \mathrm{mg} / \mathrm{kg}$.

Weinbroum (12) evaluated the effects of postoperative coadministration of small doses of ketamine $(0.250 \mathrm{mg} / \mathrm{kg})$ and morphine on pain intensity in surgical patients who complained of pain of at least $6 / 10$ on a VAS despite more than $0.1 \mathrm{mg} / \mathrm{kg}$ of IV morphine administration within 30 minutes. This study demonstrated that IV administration of a combined small dose of MK promptly and satisfactorily resolved pain that had been unresponsive to IV morphine, whereas a threefold dose of MS only partially attenuated it. Pain intensity was still less in the former group after two hours (12).

Abbasi et al. (20) compared morphine $0.1 \mathrm{mg} / \mathrm{kg}$ and placebo (MP group) and morphine $0.1 \mathrm{mg} / \mathrm{kg}$ and ketamine $0.15 \mathrm{mg} / \mathrm{kg}$ (MK group) in patients with severe renal colic pain. Assessment of the average pain during 120 minutes at $10^{\text {th }}$ and $30^{\text {th }}$ minutes after the start of the drug, MK group was significantly lower than the MP group. They showed that, given that combinations of morphine with low doses of ketamine in patients with renal colic pain causes more pain and morphine consumption reduction.

Several studies have also compared the analgesic effects and safety of ketamine in pre-hospital. Jennings et al. (21) in a systematic review of the effects of ketamine on reducing pain in pre-hospital trauma showed that ketamine is a safe and effective analgesic agent. The addition of ketamine as an analgesic agent may improve the management of patients presenting with acute traumatic pain in the pre-hospital setting. A systematic review, too, showed that the use of ketamine as a supplementary medication results in a decrease in the need for morphine, preventing unfavorable complications (22).

In our study, all adverse effects were weak and brief because no one needed treatment and patients' satisfaction was not different between the two groups. There was no clinically significant change of vital signs after enrolment, nor were there any clinically significant differences between study groups. These findings are consistent with those of previous trials of ketamine and opioid combination regimens (11-19). Jennings et al. (21) reported similar findings but emphasized that more minor complications are seen with a combination of morphine/ketamine.

The follow-up period of our trial is a limitation because the patients were observed for 60 minutes. Increasing patients' follow-up time provided the possibility of obtaining valuable results. Indeed, Weinbroum (12) demonstrated that the analgesic effects of the ketamine group were clearly evident throughout the 120-minute observation period.

\section{Conclusion}

This study showed that adding low-dose ketamine $(0.3 \mathrm{mg} / \mathrm{kg})$ to low-dose morphine $(0.05 \mathrm{mg} / \mathrm{kg})$ in the MK group results in a significant decrease in the severity of acute pain in trauma patients and significantly reduced the need for additional dose of morphine without increasing complications; whereas, the difference in pain reduction between the two groups was not significant. We demonstrated that combinations of low-dose morphine and low-dose ketamine is an effective and safe method of pain control in trauma patients.

\section{Acknowledgment}

The authors would like to acknowledge Azadeh Javaherpour from McGill University, Montreal, Quebec, Canada for her collaboration in this study.

\section{Ethics}

Ethics Committee Approval: The study protocol was approved by the ethics committee of Isfahan University of Medical Sciences (IR.MUI.REC.1395.3.824). The trial was registered in the Iranian Registry of Clinical Trials under the number (IRCT20180129038549N3).

Informed Consent: Before the study, written informed consent was obtained from all parents, before enrolment into the study.

Peer-review: Externally and internally peer-reviewed.

\section{Authorship Contributions}

Surgical and Medical Practices: F.H., R.A., B.M., A.A.G., Concept: F.H., R.A., Design: F.H., R.A., B.M., A.A.G., Data Collection or Processing: F.H., R.A., B.M., A.A.G., Analysis or Interpretation: F.H., R.A., Literature Search: F.H., R.A., B.M., A.A.G., Writing: F.H., R.A., B.M., A.A.G.

Conflict of Interest: No conflict of interest was declared by the authors.

Financial Disclosure: The authors declared that this study received no financial support.

\section{References}

1. Scholten AC, Berben SA, Westmaas AH, Van Grunsven PM, de Vaal ET, Rood PP, et al. Pain management in trauma patients in (pre) hospital based emergency care: current practice versus new guideline. Injury. 2015;46:798806.

2. Berben SA, Meijs TH, van Grunsven PM, Schoonhoven L, van Achterberg T. Facilitators and barriers in pain management for trauma patients in the chain of emergency care. Injury. 2012;43:1397-402. 
3. Jahanian F, Hosseininejad SM, Ahidashti HA, Bozorgi F, Khatir IG, Montazar $\mathrm{SH}$, et al. Efficacy and safety of morphine and low dose ketamine for pain control of patients with long bone fractures: a randomized, double-blind, clinical trial. Bull Emerg Trauma. 2018;6:31-6.

4. Häske D, Böttiger BW, Bouillon B, Fischer M, Gaier G, Gliwitzky B, et al. Analgesia in patients with trauma in emergency medicine: a systematic review and meta-analysis. Deutsches Ärzteblatt Int. 2017;114:785-92.

5. Ahmadi A, Bazargan-Hejazi S, Zadie ZH, Euasobhon P, Ketumarn P, Karbasfrushan A, et al. Pain management in trauma: A review study. J Inj Violence Res. 2016;8:89-98.

6. Gausche-Hill M, Brown KM, Oliver ZJ, Sasson C, Dayan PS, Eschmann NM, et al. An evidence-based guideline for prehospital analgesia in trauma. Prehosp Emerg Care. 2014;18(Supl 1):25-34.

7. Malchow RJ, Black IH. The evolution of pain management in the critically ill trauma patient: emerging concepts from the global war on terrorism. Crit Care Med. 2008;36:S346-57.

8. Masoumi B, Farzaneh B, Ahmadi O, Heidari F. Effect of intravenous morphine and ketorolac on pain control in long bones fractures. Adv Biomed Res. 2017;6.91.

9. Bijur PE, Kenny MK, Gallagher EJ. Intravenous morphine at $0.1 \mathrm{mg} / \mathrm{kg}$ is not effective for controlling severe acute pain in the majority of patients. Ann Emergency Med. 2005;46:362-7. [Epub 2005/09/29].

10. Heydari F, Gholamian A, Zamani M, Majidinejad S. Effect of intramuscular ketamine versus haloperidol on short-term control of severe agitated patients in emergency department: a randomized clinical trial. Bull Emerg Trauma. 2018;6:292-9.

11. Kapfer B, Alfonsi P, Guignard B, Sessler DI, Chauvin M. Nefopam and ketamine comparably enhance postoperative analgesia. Anesth Analg. 2005;100:16974.

12. Weinbroum AA. A single small dose of postoperative ketamine provides rapid an sustained improvement in morphine analgesia in the presence of morphine-resistant pain. Anesth Analg. 2003;96:789-95.
13. Sin B, Ternas T, Motov SM. The use of subdissociative-dose ketamine for acute pain in the emergency department. Acad Emerg Med. 2015;22:251-7.

14. Galinski M, Dolveck F, Combes X, Limoges V, Smail N, Pommier V, et al. Management of severe acute pain in emergency settings: ketamine reduces morphine consumption. Am J Emerg Med. 2007;25:385-90.

15. Farsi D, Movahedi M, Hafezimoghadam P, Abbasi S, Shahlaee A, RahimiMovaghar V. Acute pain management with intravenous $0.10 \mathrm{mg} / \mathrm{kg}$ vs. 0.15 $\mathrm{mg} / \mathrm{kg}$ morphine sulfate in limb traumatized patients: a randomized doubleblinded placebo-controlled trial. TJTES. 2013;19:398-404. [Epub 2013/11/12].

16. Bounes V, Charpentier S, Houze-Cerfon CH, Bellard C, Ducassé JL. Is there an ideal morphine dose for prehospital treatment of severe acute pain? A randomized, double-blind comparison of 2 doses. Am J Emerg Med. 2008;26:148-54.

17. Majidinejad S, Esmailian M, Emadi M. Comparison of intravenous ketamine with morphine in pain relief of long bones fractures: a double blind randomized clinical trial. Emergency. 2014;2:77-80.

18. Miller JP, Schauer SG, Ganem VJ, Bebarta VS. Low-dose ketamine vs morphine for acute pain in the ED: a randomized controlled trial. Am J Emerg Med. 2015;33:402-8.

19. Beaudoin FL, Lin C, Guan W, Merchant RC. Low-dose ketamine improves pain relief in patients receiving intravenous opioids for acute pain in the emergency department: results of a randomized, double-blind, clinical trial. Acad Emerg Med. 2014;21:1193-202.

20. Abbasi S, Bidi N, Mahshidfar B, Hafezimoghadam P, Rezai M, Mofidi M, et al. Can low-dose of ketamine reduce the need for morphine in renal colic? A double-blind randomized clinical trial. Am J Emerg Med. 2018;36:376-9.

21. Jennings PA, Cameron P, Bernard S. Ketamine as an analgesic in the prehospital setting: a systematic review. Acta Anaesthesiologica Scandinavica. 2011;55:638-43.

22. Subramaniam K, Subramaniam B, Steinbrook RA. Ketamine as adjuvant analgesic to opioids: a quantitative and qualitative systematic review. Anesth Analg. 2004;99:482-95. 